\title{
The Recent Advances of Magnetic Nanoparticles in Medicine
}

\author{
Ting Guo, ${ }^{1}$ Mei Lin $\mathbb{D}^{2}{ }^{2}$ Junxing Huang, ${ }^{3}$ Chenglin Zhou, ${ }^{2}$ Weizhong Tian, ${ }^{4}$ Hong Yu, ${ }^{5}$ \\ Xingmao Jiang $\mathbb{D},{ }^{6}$ Jun $Y e\left(\mathbb{D},{ }^{1}\right.$ Yujuan Shi, ${ }^{1}$ Yanhong Xiao $\mathbb{D},{ }^{1}$ Xuefeng Bian, ${ }^{1}$ \\ and Xiaoqian Feng ${ }^{1}$
}

\footnotetext{
${ }^{1}$ Institute of Clinical Medicine, Taizhou People's Hospital Affiliated to Nantong University, Taizhou, Jiangsu 225300, China

${ }^{2}$ Clinical Laboratory, Taizhou People's Hospital Affiliated to Nantong University, Taizhou, Jiangsu 225300, China

${ }^{3}$ Oncology Department, Taizhou People's Hospital Affiliated to Nantong University, Taizhou, Jiangsu 225300, China

${ }^{4}$ Imaging Department, Taizhou People's Hospital Affiliated to Nantong University, Taizhou, Jiangsu 225300, China

${ }^{5}$ Pathology Department, Taizhou People's Hospital Affiliated to Nantong University, Taizhou, Jiangsu 225300, China

${ }^{6}$ School of Chemical Engineering and Pharmacy, Wuhan Institute of Technology, Wuhan, Hubei 430000, China
}

Correspondence should be addressed to Mei Lin; 1_mei@163.com

Received 28 December 2017; Accepted 16 April 2018; Published 29 April 2018

Academic Editor: Federica Paladini

Copyright ( 2018 Ting Guo et al. This is an open access article distributed under the Creative Commons Attribution License, which permits unrestricted use, distribution, and reproduction in any medium, provided the original work is properly cited.

With the progress of nanotechnology and molecular biology, nanoparticles have been widely studied and applied in biomedicine. Particularly, characterized by unique magnetic property, targeting, and biocompatibility, magnetic nanoparticles have become one of the research hotspots in the nanomedical field. Herein, we summarized the recent advances of magnetic nanoparticles in medicine, including the property, carrier function, MRI, and tumor magnetic inductive hyperthermia of magnetic nanoparticles.

\section{Introduction}

The constant development of medicine has rendered early diagnosis and precise treatment of its development direction. Nanotechnology has provided a new platform for medicine development. As a result of unique features, nanomaterials have been extensively studied and applied in the medical field [1]. As one kind of the nanomaterials, magnetic nanoparticles possess not only the general characteristics of nanoparticles but also the magnetic properties. After being modified surfacely, magnetic nanoparticles can possess excellent biocompatibility, which is suitable for medical application. For example, surface-modified magnetic nanoparticles can be used as vectors, allowing for drug or gene directional transportation under the action of the magnetic field to realize targeted therapy [2]. Moreover, under the action of applied magnetic field, magnetic nanoparticles have unique magnetic sensitivity, which can thus be applied in MRI [3]. Furthermore, the magnetocaloric effect of magnetic nanoparticles has also provided a new means for tumor treatment [4]. All in all, the application of magnetic nanoparticles will further promote the development of the medical field.

\section{Category and Properties of Magnetic Nanoparticles}

At present, magnetic nanoparticles (NPs) mainly include metal NPs, metal oxide NPs, and metal alloy NPs. The common NPs are gold [5], silver [6], iron, cobalt, and nickel. Metal oxide NPs mainly include iron oxides $\left(\gamma-\mathrm{Fe}_{2} \mathrm{O}_{3}\right.$ and $\left.\mathrm{Fe}_{3} \mathrm{O}_{4}\right)$ and ferrites $\left(\mathrm{CoFe}_{2} \mathrm{O}_{4}\right.$ and $\left.\mathrm{Mn}_{0.6} \mathrm{Zn}_{0.4} \mathrm{Fe}_{2} \mathrm{O}_{4}\right)$, and metal alloy $\mathrm{NPs}$ cover $\mathrm{FeCo}, \mathrm{FePt}$, and so on. Of them, metal oxide $\mathrm{Fe}_{2} \mathrm{O}_{3}$ and $\mathrm{Fe}_{3} \mathrm{O}_{4}$ magnetic nanoparticles are the most extensively used magnetic NPs [7], which can be handily prepared and easily controlled in particle size and shape [8]. Some metallic elements such as manganese ( $\mathrm{Mn})$ and $\mathrm{Zn}(\mathrm{Zn})$ can be added to nanosized iron oxide structure to prepare a variety of ferrite nanoparticles $\left(\mathrm{Mn}_{3} \mathrm{Zn}_{7} \mathrm{Fe}_{2} \mathrm{O}_{4}, \mathrm{Mn}_{0.6} \mathrm{Zn}_{0.4} \mathrm{Fe}_{2} \mathrm{O}_{4}\right.$, and so on). These ferrite nanoparticles have a stronger magnetism and a higher relaxation rate, which contributes to their application for magnetic resonance imaging (MRI).

Magnetic NPs own the following properties. Firstly, magnetic nanometer materials have nonvirulence and nonimmunogenicity. Secondly, magnetic NPs possess the surface 
TABLE 1: Frequently used compounds to surfacely modify magnetic NPs.

\begin{tabular}{|c|c|c|}
\hline Modified compounds & Advantages & Applications \\
\hline PEG [12] & $\begin{array}{l}\text { Enhanced water solubility of NPs, reduced RES } \\
\text { phagocytosis, and increased blood circulation time }\end{array}$ & MRI, tumor diagnosis, and treatment \\
\hline PEI & Good biocompatibility & Gene and drug vectors \\
\hline Polyvinyl alcohol (PVA) & Elevated stability and reduced particle aggregation & MRI, vectors, and bioseparation \\
\hline Glucan & Excellent stability and extended in vivo circulation time & Drug vectors \\
\hline Chitosan [13] & Good stability and biocompatibility & Vector, thermotherapy \\
\hline Liposome [14] & Good biocompatibility & Tumor treatment, thermotherapy, and MRI \\
\hline FA [15] & $\begin{array}{l}\text { Good biocompatibility, essential small-molecule vitamin } \\
\text { for the human body }\end{array}$ & $\begin{array}{c}\text { Targeted receptors, diagnosis, and treatment } \\
\text { of tumors (breast cancer, cervical cancer, and } \\
\text { ovarian cancer) }\end{array}$ \\
\hline Gold [16, 17] & $\begin{array}{l}\text { Biocompatibility can provide optical property and } \\
\text { magnetism for biological application }\end{array}$ & Tumor diagnosis and MRI \\
\hline
\end{tabular}

effect. In detail, they have great specific surface area, which is good for carrying a large amount of DNA fragments, drugs, and modified compounds. After modification, they can be used as vector. Thirdly, most modified magnetic NPs have excellent biocompatibility. Fourthly, some magnetic NPs have superparamagnetism.

General magnetic materials are a multimagnetic domain structure. When the size of magnetic materials is reduced to nanoscale, they have a single magnetic domain structure and their magnetism turns to paramagnetism [9]. Paramagnetic materials, in the external magnetic field, are macroscopically nonmagnetic, showing a very weak magnetic property. When the size of the magnetic material is smaller than the critical size (generally $20 \mathrm{~nm}$ ), the magnetic spin of the magnetic nanomaterials will be disordered and superparamagnetic, which will be magnetized rapidly under the action of alternating magnetic field and can move in a directional manner with the magnetic field. But once the magnetic field is removed, the magnetization becomes zero, that is, it does not show magnetism when there is no external magnetic field [10]. The magnetic susceptibility of superparamagnetism in the presence of external magnetic field is much higher than that of general paramagnetic materials. Fifthly, magnetic NPs can be used for tumor thermotherapy since they can produce thermal effect under the action of alternating magnetic field (AMF). Sixthly, the magnetic NPs can be exploited for magnetic separation [11]. For instance, they can be served as vectors to bind biomolecules and then be separated from the biomolecules at the targeted area under the action of the magnetic field and thus used for targeted therapy or diagnosis. Since most PH outside tumor cells are usually low, most weak alkaline drugs can hardly enter the cells, showing drug resistance. In order to overcome such resistance, researchers have extensively studied the preparation and modification of multiple NPs. As superparamagnetic iron oxide (SPIO) NPs are being studied most extensively, $\mathrm{Fe}_{2} \mathrm{O}_{3}$ and $\mathrm{Fe}_{3} \mathrm{O}_{4}$ NPs have been widely applied in diagnosis and treatment for all kinds of tumors. Iron oxide NPs have many advantages such as excellent biodegradability, low cytotoxicity, the ability to be modified by multiple substances, the ability to bind with multiple targeted ligands or antibodies, and ease to prepare, and can enter cells through endocytosis. They have been used in MRI and tumor thermotherapy [7].

\section{Modification of Magnetic Nanoparticles}

As a result of the surface effect and interface effect of nanostructure, the exposed magnetic NPs are extremely unstable in structure and are apt to aggregation. After entering the human body, the magnetic NPs are subject to being absorbed with body proteins and phagocytosed by the vascular endothelial system. They thus cannot be applied in biomedicine. It has been confirmed that surface modification can better stabilize the nanostructure and improve the surface functionalization of nanoparticles. For instance, when coated by active material with hydroxyl and carboxyl, nanoparticles can bind DNA fragments, drugs, and proteins, thus exerting the function of transportation and targeted therapy. At present, numerous surface-modified compounds are available, including polyethylene glycol (PEG), polyethylenimine (PEI), folic acid (FA), liposome, noble metal, and inorganic materials (Table 1).

Surface modification can enhance the water solubility, biocompatibility, and stability of NPs; they thus can be served as vectors for drug delivery, gene transfer, MRI, and thermotherapy. Some surface-modified compounds such as PEG and carboxylated polyethyleneimine (PEI-COOH) have rendered favorable water solubility for magnetic NPs, leading to good application in MRI or other medical diagnosis and treatments $[18,19]$. As one of the few polymers which can be used for in vivo injection, PEG has favorable water solubility, biocompatibility, and nontoxicity. It can bind onto the surface of NPs by end-group reactivity and thus be able to bind DNA fragments, drugs, and other biological fragments, extending the blood circulation time [20, 21]. PEG-modified ferrite NPs can enter cells through phagocytosis, which can suppress iron ion release when it is degraded in lysosome, lessening the binding of iron ion with catalase to reduce cell destruction [22]. PEI is a cationic polymer, which can carry gene fragments and drugs, thus carrying out drug or gene transfer. Tang et al. used PEI to modify Mn Zn ferrite and bound it with plasmid DNA. Agarose gel electrophoresis 
(AGE) suggested a reliable binding of $\mathrm{PEI} / \mathrm{Mn}_{0.5} \mathrm{Zn}_{0.5} \mathrm{Fe}_{2} \mathrm{O}_{4} /$ DNA [23]. In the applied magnetic field, PEI-modified magnetic NPs have remarkably improved gene transfection efficiency [24]. Additionally, magnetic NPs receiving specific surface modification can specifically bind with target cell, thus being served as the vector for contrast medium of MRI or nanoprobe for the early diagnosis of tumor. Inert metal NPs also have aroused extensive attention. On the one hand, the gold nanocoating can bind with sulfhydryl-containing ligand, which can be used as vectors of biomolecules. On the other hand, the gold-coating surface can enhance its optical property and thus can be used to trace the NPs. Methacrylate- (PDEA-) coating magnetic NPs can resist the degradation of DNase I to the carried gene during the gene transfection of G2 cell hepatitis, thus improving gene transfection efficiency [25].

\section{Application of Magnetic NPs in MRI}

In recent years, tumor morbidity is increasing and early tumor diagnosis is of vital importance. MRI is one of the common methods for tumor diagnosis. With the development of nanotechnology, nanometer materials have been gradually applied for the diagnosis and treatment of tumor. Particularly, the combination of nanotechnology with MRI to detect tumor has attained satisfactory effects. In MRI, magnetic NPs have displayed unique sensitivity to the magnetic field in the presence of applied magnetic field. They can alter $\mathrm{T} 1$ or $\mathrm{T} 2$ relaxation time of MRI, which can thereby greatly enhance the diagnostic efficiency of MRI [26]. However, water solubility of magnetic NPs has to be enhanced in the meantime in preserving their magnetic property so that they can be extensively applied in clinics [27]. Research has indicated that PEG-modified NPs have excellent water solubility and biocompatibility, which can extend the blood circulation time and enhance the MRI effect [28].

The application of nanoprobe in MRI is one of research hotspots in molecular imaging field. After NPs coupled with ligands or antibodies enter the human body, they may be bound with the specific receptors or antibodies of tumor cells and thus be phagocytosed by tumor cells or bind onto the tumor cell surface, resulting in NPs aggregating in tumor tissues. Under such circumstances, magnetic nanomaterials will generate magnetism under the action of applied magnetic field, which allows for early diagnosis and detection of small lesion through MRI. MRI research on magnetic ferrite NPs coated with carboxylated PEG suggests that the MR transverse relaxation time $\mathrm{T} 2$ has reduced with the increase in $\mathrm{NP}$ concentration, while signals on MR T2-weighted image have enhanced [29].

Chitosan (CS), a surface modification of cationic polymer also can render excellent water solubility and stability for magnetic NPs. Lactobionic acid (LA) modification allows them to bind with hepatic cell surface receptor, thus being phagocytosed by hepatic cells. After superparamagnetic iron oxide NPs (CS-LA@SPION) modified with CS and LA together were injected into nude mice, MRI showed remarkably lowered T2-weighted image in the liver within $1 \mathrm{~h}$ after, which indicated they can be used as liver-targeted MRI contrast medium [30]]. Applying PEG to modify $\mathrm{Fe}_{3} \mathrm{O}_{4}$ NPs and then coupling them with chloride channel buthotoxin (CTX), Sun et al. prepared glioma nanoprobe. It was shown in MRI that such nanoprobe could effectively bind with glioma cells [31]. Another study showed that NP-PEG-CTX-Cy5.5 nanoprobe prepared by using $\mathrm{Fe}_{3} \mathrm{O}_{4}$ NPs as core and then coating the core with PEG, subsequently binding it with fluorescence molecules Cy5.5 and CTX, could not only be used for MRI of glioma but also could be detected by fluorescence microscopy [32]. Anbarasu et al. labeled the PEG-coated $\mathrm{Fe}_{3} \mathrm{O}_{4}$ NPs with monoclonal antibody and then planted it into the colon cancer mouse model. They successfully conducted targeted localization by MRI [33]. In addition, adding metallic elements such as $\mathrm{Mn}$ and $\mathrm{Zn}$ into the nanometer ferrite structure can change the properties of NPs [33], enhancing the magnetism of NPs (such as $\mathrm{Mn}_{0.5} \mathrm{Zn}_{0.5} \mathrm{Fe}_{2} \mathrm{O}_{4}$ and $\mathrm{Mn}_{0.6} \mathrm{Zn}_{0.4} \mathrm{Fe}_{2} \mathrm{O}_{4}$ ), increasing the transverse relaxation time of ferrite NPs, elevating the T2 MRI imaging contrast, darkening the T2-weighted image, and it thus can be better applied in MRI [34].

Stem cell has attracted extensive attention in research on biomedicine owing to its excellent proliferative capacity and differentiation potential. In this way, an effective, nontoxic and stable cell labeling is required to better investigate the treatment mechanism of stem cell and to monitor differentiation and migration of stem cells. Currently, there are two methods to label stem cells using the superparamagnetic NPs. One is cell surface labeling and the other one is intracellular labeling. It was discovered that cell surface labeling is likely to be eliminated by the reticuloendothelial system (RES). In comparison, intracellular labeling has certain requirements on the concentration of NPs. Its effective safe concentration was $20-25 \mathrm{mg} / \mathrm{L}$ [35]. In the glioma mouse experiment, after superparamagnetic NP-labeled stem cells were injected into the mouse, labeled stem cell migration could be observed in the mouse under MRI within 10 days after injection. Furthermore, cells injected into the tumor tissue were identified histologically to be the superparamagnetic NP-labeled stem cells. The function and activity of these cells are not affected, suggesting that superparamagnetic NPs can be used for labeling stem cells [36].

Currently, most research on targeted therapy for tumor is based on single point. But the therapy effect of most targeted therapy based on single point is poor. Therefore, it remains a challenge to research and develop multitarget therapy for tumor cell, so as to further improve the efficacy of such therapy on tumor. As we all know, tumor tissue is associated with exuberant angiogenesis. Consequently, combined application of targeted magnetic induction thermotherapy targeting tumor cell and tumor vascular hyperplasia endothelial cell may contribute to enhancing the efficacy. Additionally, some genes overexpress in multiple tumors. For instance, CD44 excessively expresses in tumors in the reproductive system, digestive system, and respiratory system. If general targeted molecular probe is designed, the development of research in the field of tumor molecular imaging diagnosis may be greatly promoted. However, there is still a long way to go for developing a general gene-targeted probe due to the mutagenicity of tumor cell. 


\section{Research on Magnetic NPs as Vectors}

Gene therapy is a treatment by transferring the exogenous gene or gene fragment into the target gene of patient [37]. It is another novel treatment for tumor after traditional surgery, radiotherapy, and chemotherapy [38]. But gene transfer method with safety, efficiency, and controllability is the key for gene therapy [39]. DNA is extremely unstable inside and outside the cell and is likely to be degraded by nucleotidase. Stably transferring exogenous gene into target cell for gene therapy depends on the gene transfer system [40], and stable and effective gene vector is of importance to gene therapy [41].

Gene vectors can be classified into two types, the viral and nonviral vectors. Viral gene vectors, such as adenovirus, herpes simplex virus, and smallpox virus [42], can transfer the target gene into cell by viral vector for gene expression [43]. Viral vector is linked with high transfection efficiency. However, it has the drawbacks of immune response, limited number of genes carried by virus, unavoidable random insertion of viral vector into the host chromosome during gene transfer, and high expenses [44]. In Europe, one gene therapy using viral vector has been applied in clinics, but its treatment expenses are as high as 1 million dollars [45]. All these unfavorable factors have restricted the extensive application of viral vector. Therefore, nonviral gene vector research has attracted wide attentions.

The common nonviral gene vectors include cationic liposome, cationic polymer, and NPs. Liposome and polymer are two gene vectors that are extensively applied. The structure and size of cationic liposome binding with DNA are related to its transfection efficiency [46]. Most nonviral gene vectors have low transfection efficiency, which lack targeting. PEI is the common cationic liposome, and its positive charge can absorb DNA, thus forming the structure similar to core-shell, which can maintain DNA stability.

Nanocarriers have attracted increasing attention with the booming of nanotechnology. NPs can be easily modified and have excellent biocompatibility and little immune response. They are likely to enter tissue after coupling with related ligands or antibodies, thus binding with the cell surfacespecific receptors or specific antigens. Alternatively, they can be phagocytosed by target cell and thereby enter the cell to realize DNA transfer. Studies have shown that some nanocarriers modified specifically have high gene transfer efficiency [47]. Magnetic NPs can carry out directional movement under the action of AMF, thereby enhancing the transfer efficiency. Moreover magnetic NPs can be used as vectors to bind with biomolecules by magnetic separation. These biomolecules can separate from vectors under the action of applied magnetic field, thus contributing to achieve the goal of targeted therapy.

It was discovered that doxorubicin magnetic NPs (DOX MNPs) can remarkably increase DOX uptake by glioma cells in the magnetic field. MTT assay revealed that death rate of tumor cells after DOX MNP uptake is notably higher than that after DOX uptake only [48].The Fe3O4 @Alg-GA NPs synthesized and modified by sodium alginate and D-galactosamine can enhance their uptake by human hepatoma cells under the action of AMF. Moreover, they can produce the heating effect and kill $95 \%$ cells [49]. A research on pancreatic cancer showed that chemotherapeutic DOX can be rapidly transferred and released in pancreatic cancer cells by using superparamagnetic iron oxide (SPIO) NPs as the vectors monitored under electron microscope, $\mathrm{X}$-ray, and optical microscope [50]. As we all know, most chemotherapeutics cannot pass the blood brain barrier (BBB). But a novel administration route has been discovered in brain tumor animal experiment. When paclitaxel- and curcumin-targeted therapies were carried out in glioma mouse using the DL-lactic acid-glycolic acid (PLGA) magnetic NPs, the chemotherapeutics could rapidly pass the $\mathrm{BBB}$ by the vectors [51]. In a research on colon tumor, PEG was applied in modifying the uperparamagnetic ferrite core and then connected with the amino terminal fragment hATF of human recombinant protein, forming the targeted probe hATF-SPIO with the diameter of about $30 \mathrm{~nm}$. It was demonstrated that the probe can specifically bind with uPARexpressing tumor cells in vitro and develop in a targeted manner in the colon tumor animal models with moderately expressed UPAR [52]. With the development of nanosensor technology, hepatitis viruses in human blood can be quantitatively detected [53]. In the quantitative detection of tumor markers, immunosensor has considerable potential in the early detection of tumor [54].

With regard to the toxicity of magnetic NPs, many studies demonstrate that most superparamagnetic NPs have excellent biocompatibility. But some other studies indicate that NPs have toxicity to neurons and glial cells and the toxicity is related to the compounds modified on the surface of magnetic NPs [55].

\section{Tumor-Targeted Thermotherapy of Magnetic Nanometer Materials}

Thermotherapy is a method to kill tumor cells with thermal energy at a certain temperature. It can be applied alone and also can be used in combination with other therapies such as surgery, radiotherapy, and chemotherapy, exerting good synergistic effect [56]. It is reported that the therapeutic efficacy of paclitaxel can increase by $10-100$ folds at $43^{\circ} \mathrm{C}$ for $30 \mathrm{~min}$. In addition, the killing capacity of chemotherapeutics with low cytotoxicity under normal temperature can be doubled after heated [57]. Jordan et al. [58] first applied magnetic NPs in tumor thermotherapy and invented magnetic fluid thermotherapy (MFH). Magnetic fluid is a liquid magnetic material, which possesses magnetic properties as well as water solubility. It is appropriate for clinical application. Under an AMF, the nanoparticle core of magnetic fluid can transform the magnetic energy into thermal energy, which can stably elevate the temperature of tumor tissue, thus inhibiting tumor growth or killing tumor cell or inducing tumor cell apoptosis [59-61].

Generally, tumor tissue is associated with rapid angiogenesis, which may result in incomplete structure of the capillary wall, disordered branch and distorted structure and fragility. With the addition of tumor compression, the above factors may lead to circulation blocking. Moreover, during 
magnetic thermotherapy for tumor, thermal dissipation is slow in tumor cells, which together with circulation blocking may give rise to local anaerobic metabolism. Consequently, the cancer cells are more sensitive to heat than normal cells [62]. Thermotherapy may kill tumor cells by reducing vascular endothelial cell regeneration to destroy vascular structure [63], reducing enzyme system activity on the tumor cell membrane, destroying mitochondrion resulting in energy supply disorder [64], inhibiting the activities of DNA polymerase and ligase in tumor cells resulting in DNA and RNA synthesis being disordered and regulating the expression of apoptosis-related genes to induce cell apoptosis [65]. Temperature at $42-45^{\circ} \mathrm{C}$ is considered to be the appropriate temperature for tumor thermotherapy, which can kill tumor cells, with no injury to normal tissues.

The magnetism of magnetic fluid depends on magnetic particles, among which $\mathrm{Fe}_{3} \mathrm{O}_{4}$ is the most extensively used magnetic fluid material. The magnetism of $\mathrm{Fe}_{3} \mathrm{O}_{4}$ nanoparticles can be enhanced by adding some metallic elements such $\mathrm{Mn}$ and $\mathrm{Zn}$ during preparation. By adjusting the proportion of $\mathrm{Mn}$ and $\mathrm{Zn}$, the temperature-sensitive $\mathrm{Mn}_{\mathrm{x}} \mathrm{Zn}_{1-\mathrm{x}} \mathrm{Fe}_{2} \mathrm{O}_{4}$ (Mn Zn ferrite) invented in Southeast University can transform magnetic energy into thermal energy below the Curie temperature under the action of AMF, thus elevating the temperature. But when the temperature arrives at the Curie temperature, it becomes a nonmagnetic material, stopping absorbing magnetic energy, thus decreasing the temperature. So cyclically, the temperature always maintains around the Curie temperature [66]. In this way, such $\mathrm{Mn} \mathrm{Zn}$ ferrite NPs are endowed with the self-controlled temperature ability. Moreover, the Mn Zn ferrite NPs have numerous advantages during magnetic thermotherapy, such as thermal bystander effect, universality, high-characteristic absorption rate, constant temperature, and excellent biocompatibility. MFH can effectively destroy cancer cells, with no injury to normal cells. It is considered as one of the most promising cancer therapies. A study on the safety of $\mathrm{Mn} \mathrm{Zn}$ ferrite NPs indicated that the hemolytic rate of Mn Zn ferrite magnetic fluid was $1.0429 \%$ and the $\mathrm{LD}_{50}$ was $7.186 \mathrm{~g} / \mathrm{kg}$. The bone marrow micronucleus formation rate had no significant difference between the experimental group and the negative control group [67].

It is discovered in a hepatoma cell research that when the constructed P[5HRE] AFPp-p53/PEI-Fe304 magnetic NPs were used to mediate gene therapy combined with $\mathrm{MFH}$, they could distinctly suppress the proliferative activity of hepatoma HepG2 cells. The effect was markedly superior to that in control the NP group and negative control group [68]. Xie et al. [69] modified Mn $\mathrm{Zn}$ ferrite NPs $\left(\mathrm{Mn}_{0.5} \mathrm{Zn}_{0.5} \mathrm{Fe}_{2} \mathrm{O}_{4}\right)$ with PEG lipid molecules through hydrophobic interaction. Such nanoparticles with core-shell structure rendered high magnetism, strong alternating magnetothermal effect, and good biocompatibility. In the early symptom liver metastasis mouse model of colorectal cancer, the oleic acid-wrapped ferrite RGF polypeptide complex $\left(\mathrm{Fe}_{3} \mathrm{O}_{4} @ P M A O \_\mathrm{RGD}\right)$ was injected into the hepatic artery to bind with the surface $\alpha \mathrm{V} \beta 3$ receptor of the tumor cell and then $\mathrm{MFH}$ was conducted under the action of AMF. It was found that the activity of colorectal cancer liver metastasis tumor cells was remarkably lowered [70]. Combined therapy of $\mathrm{MFH}$ with targeted chemotherapy and (or) gene therapy had shown a promising application prospect in the diagnosis and treatment for tumor. Using $\mathrm{Mn} 0.5 \mathrm{Zn} 0.5 \mathrm{Fe} 2 \mathrm{O} 4$ as vector, Lin et al. combined radionuclide, suicide gene, and $\mathrm{MFH}$ organically to treat liver cancer. The results indicated an excellent effect, and the tumor inhibition rate of the combined therapy group was remarkably higher than that of any other single therapy [71]. Another research showed that magnetic $\mathrm{CAs}_{2} \mathrm{O}_{3} / \mathrm{Fe}_{2} \mathrm{O}_{3}$ nanocomposites adopted to thermochemotherapy for hepatoma had an evident higher effect than that of the single thermotherapy and the $\mathrm{As}_{2} \mathrm{O}_{3}$ chemotherapy alone and the equivalent dosage of chemotherapeutics was greatly lowed and has few side effects [72].

\section{Problem and Prospect}

With the development of nanoscience and nanotechnology, the research and application of magnetic NPs have made considerable progress in the biomedical field. For example, magnetic NPs have been extensively applied in biomolecule vectors, targeted localization, MRI, and thermotherapy. However, most studies remain at the laboratory research stage, and few have really been applied in clinics. Their toxicity, side effects, long-term efficacy, and in vivo metabolic mechanism still need to be further studied. A large amount of basic and clinical research needs to be done for their clinical application. Confidently, magnetic NPs will have a broad application prospect in the medical field, with the increasing development and the further intensive research of nanotechnology, biotechnology, and medicine.

\section{Conflicts of Interest}

The authors declare that there is no conflict of interests.

\section{Acknowledgments}

This work is financially supported by the National Natural Science Foundation of China (81571797), the 333 Plan Foundation of Jiangsu, China (BRA2017173), and the project of Taizhou People's Hospital (ZL201734).

\section{References}

[1] R. Saini, S. Saini, and S. Sharma, "Nanotechnology: the future medicine," Journal of Cutaneous and Aesthetic Surgery, vol. 3, no. 1, pp. 32-33, 2010.

[2] T. Eslaminejad, S. N. Nematollahi-Mahani, and M. Ansari, "Glioblastoma targeted gene therapy based on pEGFP/p53loaded superparamagnetic iron oxide nanoparticles," Current Gene Therapy, vol. 17, no. 1, pp. 59-69, 2017.

[3] Y.X. J. Wang, S. M. Hussain, and G. P. Krestin, "Superparamagnetic iron oxide contrast agents: physicochemical characteristics and applications in MR imaging," European Radiology, vol. 11, no. 11, pp. 2319-2331, 2001.

[4] P. Xue, L. Sun, Q. Li et al., "PEGylated polydopamine-coated magnetic nanoparticles for combined targeted chemotherapy and photothermal ablation of tumour cells," Colloids and Surfaces B: Biointerfaces, vol. 160, pp. 11-21, 2017. 
[5] B. D. Chithrani, A. A. Ghazani, and W. C. W. Chan, "Determining the size and shape dependence of gold nanoparticle uptake into mammalian cells," Nano Letters, vol. 6, no. 4, pp. 662-668, 2006.

[6] M. Jeyaraj, G. Sathishkumar, G. Sivanandhan et al., "Biogenic silver nanoparticles for cancer treatment: an experimental report," Colloids and Surfaces B: Biointerfaces, vol. 106, pp. 86-92, 2013.

[7] Q. A. Pankhurst, J. Connolly, S. K. Jones, and J. Dobson, "Applications of magnetic nanoparticles in biomedicine," Journal of Physics D: Applied Physics, vol. 36, no. 13, pp. R167-R181, 2003.

[8] A. Akbarzadeh, M. Samiei, and S. Davaran, "Magnetic nanoparticles: preparation, physical properties, and applications in biomedicine," Nanoscale Research Letters, vol. 7, no. 1, pp. 144-156, 2012.

[9] S. Blundell and D. Thouless, "Magnetism in condensed matter," American Journal of Physics, vol. 71, no. 1, pp. 9495, 2003

[10] J. H. Kim, S. M. Kim, and Y. I. Kim, "Properties of magnetic nanoparticles prepared by co-precipitation," Journal of Nanoscience and Nanotechnology, vol. 14, no. 11, pp. 87398744, 2014.

[11] L. Borlido, A. M. Azevedo, A. C. A. Roque, and M. R. Aires-Barros, "Magnetic separations in biotechnology," Biotechnology Advances, vol. 31, no. 8, pp. 1374-1385, 2013.

[12] D. Jin, X. Jiang, X. Jing, and Z. Ou, "Effects of concentration, head group, and structure of surfactants on the degradation of phenanthrene," Journal of Hazardous Materials, vol. 144, no. 1-2, pp. 215-221, 2007.

[13] H. Jiang, H. Wu, Y. L. Xu, J. Z. Wang, and Y. Zeng, "Preparation of galactosylated chitosan/tripolyphosphate nanoparticles and application as a gene carrier for targeting SMMC7721 cells," Journal of Bioscience and Bioengineering, vol. 111, no. 6, pp. 719-724, 2011.

[14] P. Pradhan, J. Giri, F. Rieken et al., "Targeted temperature sensitive magnetic liposomes for thermo-chemotherapy," Journal of Controlled Release, vol. 142, no. 1, pp. 108-121, 2010.

[15] D. Chen, Q. Tang, W. Xue, J. Xiang, L. Zhang, and X. Wang, "The preparation and characterization of folate-conjugated human serum albumin magnetic cisplatin nanoparticles," Journal of Biomedical Research, vol. 24, no. 1, pp. 26-32, 2010.

[16] M. Kumagai, T. K. Sarma, H. Cabral et al., "Enhanced in vivo magnetic resonance imaging of tumors by PEGylated ironoxide-gold core-shell nanoparticles with prolonged blood circulation properties," Macromolecular Rapid Communications, vol. 31, no. 17, pp. 1521-1528, 2010.

[17] I. Y. Goon, L. M. H. Lai, M. Lim, P. Munroe, J. J. Gooding, and R. Amal, "Fabrication and dispersion of gold-shell-protected magnetite nanoparticles: systematic control using polyethyleneimine," Chemistry of Materials, vol. 21, no. 4, pp. 673-681, 2009.

[18] M. Corti, A. Lascialfari, M. Marinone et al., "Magnetic and relaxometric properties of polyethylenimine-coated superparamagnetic MRI contrast agents," Journal of Magnetism and Magnetic Materials, vol. 320, no. 14, pp. e316-e319, 2008.

[19] Y. Zhang, L. Zhang, X. Song et al., "Synthesis of superparamagnetic iron oxide nanoparticles modified with MPEG-PEI via photochemistry as new MRI contrast agent," Journal of Nanomaterials, vol. 2015, Article ID 417389, 6 pages, 2015.
[20] E. C. Gryparis, M. Hatziapostolou, E. Papadimitriou, and K. Avgoustakis, "Anticancer activity of cisplatin-loaded PLGA-mPEG nanoparticles on LNCaP prostate cancer cells," European Journal of Pharmaceutics and Biopharmaceutics, vol. 67, no. 1, pp. 1-8, 2007.

[21] Z. Tu, B. Zhang, G. Yang et al., "Synthesis of poly(ethylene glycol) and poly(vinyl pyrrolidone) co-coated superparamagnetic iron oxide nanoparticle as a $\mathrm{pH}$-sensitive release drug carrier," Colloids and Surfaces A: Physicochemical and Engineering Aspects, vol. 436, pp. 854-861, 2013.

[22] M. Yu, S. Huang, K. J. Yu, and A. M. Clyne, "Dextran and polymer polyethylene glycol (PEG) coating reduce both 5 and 30 $\mathrm{nm}$ iron oxide nanoparticle cytotoxicity in $2 \mathrm{D}$ and $3 \mathrm{D}$ cell culture," International Journal of Molecular Sciences, vol. 13, no. 5, pp. 5554-5570, 2012.

[23] Q.-S. Tang, D.-S. Zhang, N. Gu, X.-M. Cong, M.-L. Wan, and L.-Q. Jin, "Synthesis and in vitro study of PEI-coated Mn-Zn ferrite: a novel gene vector," Journal of Functional Materials, vol. 38, no. 8, pp. 1268-1272, 2007.

[24] Y. B. Zhou, Z. Tang, C. Shi, S. Shi, Z. Qian, and S. Zhou, "Polyethylenimine functionalized magnetic nanoparticles as a potential non-viral vector for gene delivery," Journal of Materials Science: Materials in Medicine, vol. 23, no. 11, pp. 2697-2708, 2012.

[25] H. W. Sun, X. Zhu, L. Zhang et al., "PDEA-coated magnetic nanoparticles for gene delivery to Hep G2 cells," Biotechnology and Bioprocess Engineering, vol. 18, no. 4, pp. 648-654, 2013.

[26] H. Shokrollahi, A. Khorramdin, and G. Isapour, "Magnetic resonance imaging by using nano-magnetic particles," Journal of Magnetism and Magnetic Materials, vol. 369, no. 11, pp. 176-183, 2014.

[27] X. Y. Shi, S. H. Wang, S. D. Swanson et al., "Dendrimerfunctionalized shell-crosslinked iron oxide nanoparticles for in-vivo magnetic resonance imaging of tumors," Advanced Materials, vol. 20, no. 9, pp. 1671-1678, 2008.

[28] Z. Li, L. Wei, M. Y. Gao, and H. Lei, "One-pot reaction to synthesize biocompatible magnetite nanoparticles," Advanced Materials, vol. 17, no. 8, pp. 1001-1005, 2005.

[29] A. H. Rezayan, M. Mousavi, S. Kheirjou, G. Amoabediny, M. S. Ardestani, and J. Mohammadnejad, "Monodisperse magnetite $\left(\mathrm{Fe}_{3} \mathrm{O}_{4}\right)$ nanoparticles modified with water soluble polymers for the diagnosis of breast cancer by MRI method," Journal of Magnetism and Magnetic Materials, vol. 420, pp. 210-217, 2016.

[30] G. Jin, D. Li, L. Xia, Y. Long, Y. Liu, and J. Quan, "Preparation of chitosan-lactose acid-SPION and its application in MR imaging in vivo," Journal of Medical Science Yanbian University, vol. 37, no. 4, pp. 268-270, 2014.

[31] C. Sun, O. Veiseh, J. Gunn et al., "In vivo MRI detection of gliomas by chlorotoxin-conjugated superparamagnetic nanoprobes," Small, vol. 4, no. 3, pp. 372-379, 2008.

[32] O. Veiseh, C. Sun, J. Gunn et al., "Optical and MRI multifunctional nanoprobe for targeting gliomas," Nano Letters, vol. 5, no. 6, pp. 1003-1008, 2005.

[33] M. Anbarasu, M. Anandan, E. Chinnasamy, V. Gopinath, and K. Balamurugan, "Synthesis and characterization of polyethylene glycol (PEG) coated $\mathrm{Fe}_{3} \mathrm{O}_{4}$ nanoparticles by chemical co-precipitation method for biomedical applications," Spectrochimica Acta Part A: Molecular and Biomolecular Spectroscopy, vol. 135, no. 25, pp. 536-539, 2015. 
[34] H.-J. Chen, Z. H. Zhang, L. J. Luo, and S. Z. Yao, "Surfaceimprinted chitosan-coated magnetic nanoparticles modified multi-walled carbon nanotubes biosensor for detection of bovine serum albumin," Sensors and Actuators B: Chemical, vol. 163, no. 1, pp. 76-83, 2012.

[35] I. K. Ko, H. T. Song, E. J. Cho, E. S. Lee, Y. M. Huh, and J. S. Suh, "In vivo MR imaging of tissue-engineered human mesenchymal stem cells transplanted to mouse: a preliminary study," Annals of Biomedical Engineering, vol. 35, no. 1, pp. 101-108, 2007.

[36] S. J. Kim, B. Lewis, M. S. Steiner, U. V. Bissa, C. Dose, and J. A. Frank, "Superparamagnetic iron oxide nanoparticles for direct labeling of stem cells and in vivo MRI tracking," Contrast Media \& Molecular Imaging, vol. 11, no. 1, pp. 55-64, 2016.

[37] G. K. Y. Wong and A. T. Chiu, "Gene therapy, gene targeting and induced pluripotent stem cells: applications in monogenic disease treatment," Biotechnology Advances, vol. 28, no. 6, pp. 715-724, 2010.

[38] C. Fu, L. Lin, H. Shi et al., "Hydrophobic poly (amino acid) modified PEI mediated delivery of rev-casp-3 for cancer therapy," Biomaterials, vol. 33, no. 18, pp. 4589-4596, 2012.

[39] D. W. Pack, A. S. Hoffman, S. Pun, and P. S. Stayton, "Design and development of polymers for gene delivery," Nature Reviews Drug Discovery, vol. 4, no. 7, pp. 581-593, 2005.

[40] G. Xiaoxiao, D. Baoji, L. Yunhui, G. Ying, L. Dan, and W. Erkang, "Vectors based on nanomaterials for gene delivery," Progress in Chemistry, vol. 27, no. 8, pp. 1093-1101, 2015.

[41] L. Naldini, "Gene therapy returns to centre stage," Nature, vol. 526, no. 7573, pp. 351-360, 2015.

[42] C.-Y. Chen, C.-Y. Lin, G.-Y. Chen, and Y. C. Hu, "Baculovirus as a gene delivery vector: recent understandings of molecular alterations in transduced cells and latest applications," Biotechnology Advances, vol. 29, no. 6, pp. 618-631, 2011.

[43] S. Huang and M. Kamihira, "Development of hybrid viral vectors for gene therapy," Biotechnology Advances, vol. 31, no. 2, pp. 208-223, 2013.

[44] J. R. Viola, S. El-Andaloussi, I. I. Oprea, and C. I. E. Smith, "Non-viral nanovectors for gene delivery: factors that govern successful therapeutics," Expert Opinion on Drug Delivery, vol. 7, no. 6, pp. 721-735, 2010.

[45] C. Morrison, "\$1-million price tag set for Glybera gene therapy," Nature Biotechnology, vol. 33, no. 3, pp. 217-218, 2015.

[46] T. A. Balbino, A. A. M. Gasperini, C. L. P. Oliveira, A. R. Azzoni, L. P. Cavalcanti, and L. G. de la Torre, "Correlation of the physicochemical and structural properties of pDNA/ cationic liposome complexes with their in vitro transfection," Langmuir, vol. 28, no. 31, pp. 11535-11545, 2012.

[47] J. Hewinson, J. F. R. Paton, and S. Kasparov, "Viral gene delivery: optimized protocol for production of high titer lentiviral vectors," Methods in Molecular Biology, vol. 998, pp. 65-75, 2013.

[48] I. Venugopal, S. Pernal, A. Duproz, J. Bentley, H. Engelhard, and A. Linninger, "Magnetic field-enhanced cellular uptake of doxorubicin loaded magnetic nanoparticles for tumor treatment," Materials Research Express, vol. 3, no. 9, 2016.

[49] S. Liao, C. Liu, B. P. Bastakoti et al., "Functionalized magnetic iron oxide/alginate core-shell nanoparticles for targeting hyperthermia," International Journal of Nanomedicine, vol. 10, pp. 3315-3328, 2015.
[50] M. P. Arachchige, S. S. Laha, A. R. Naik, K. T. Lewis, R. Naik, and B. P. Jena, "Functionalized nanoparticles enable tracking the rapid entry and release of doxorubicin in human pancreatic cancer cells," Micron, vol. 92, pp. 25-31, 2017.

[51] Y. Cui, M. Zhang, F. Zeng, H. Jin, Q. Xu, and Y. Huang, "Dualtargeting magnetic PLGA nanoparticles for codelivery of paclitaxel and curcumin for brain tumor therapy," ACS Applied Materials \& Interfaces, vol. 8, no. 47, pp. 3215932169, 2016.

[52] S. Zhang, L. Wang, L. Chen et al., "Molecular image of superparamagnetic iron oxide nanopariticle labeled with hATF in colon tumor models," Journal of Biomedical Engineering, vol. 32, no. 5, pp. 1067-1074, 2015.

[53] W. Chakraborty, R. Ray, N. Samanta, and C. RoyChaudhuri, "Quantitative differentiation of multiple virus in blood using nanoporous silicon oxide immunosensor and artificial neural network," Biosensors and Bioelectronics, vol. 98, pp. 180-188, 2017.

[54] H. Afsharan, F. Navaeipour, B. Khalilzadeh et al., "Highly sensitive electrochemiluminescence detection of p53 protein using functionalized Ru-silica nanoporous@gold nanocomposite," Biosensors and Bioelectronics, vol. 80, pp. 146-153, 2016.

[55] C. Costa, F. Brandão, M. J. Bessa et al., "In vitro cytotoxicity of superparamagnetic iron oxide nanoparticles on neuronal and glial cells. Evaluation of nanoparticle interference with viability tests," Journal of Applied Toxicology, vol. 36, no. 3, pp. 361-372, 2016.

[56] M. Lin, J. X. Huang, J. Zhang et al., "The therapeutic effect of PEI- $\mathrm{Mn}_{0.5} \mathrm{Zn}_{0.5} \mathrm{Fe}_{2} \mathrm{O}_{4}$ nanoparticles/pEgr1-HSV-TK/GCV associated with radiation and magnet-induced heating on hepatoma," Nanoscale, vol. 5, no. 3, pp. 991-1000, 2013.

[57] Y. Tang and A. J. McGoron, "Increasing the rate of heating: a potential therapeutic approach for achieving synergistic tumour killing in combined hyperthermia and chemotherapy," International Journal of Hyperthermia, vol. 29, no. 2, pp. 145-155, 2013.

[58] A. Jordan, P. Wust, R. Scholz et al., "Cellular uptake of magnetic fluid particles and their effects on human adenocarcinoma cells exposed to AC magnetic fields in vitro," International Journal of Hyperthermia, vol. 12, no. 6, pp. 705-722, 1996.

[59] W. Chen, P. Yi, Y. Zhang, L. Zhang, Z. Deng, and Z. Zhang, "Composites of aminodextran-coated $\mathrm{Fe}_{3} \mathrm{O}_{4}$ nanoparticles and graphene oxide for cellular magnetic resonance imaging," ACS Applied Materials \& Interfaces, vol. 3, no. 10, pp. 40854091, 2011.

[60] K. Hayashi, K. Ono, H. Suzuki et al., "High-frequency, magnetic-field-responsive drug release from magnetic nanoparticle/organic hybrid based on hyperthermic effect," ACS Applied Materials \& Interfaces, vol. 2, no. 7, pp. 1903-1911, 2010.

[61] S. Laurent, S. Dutz, U. O. Häfeli, and M. Mahmoudi, "Magnetic fluid hyperthermia: focus on superparamagnetic iron oxide nanoparticles," Advances in Colloid and Interface Science, vol. 166, no. 1-2, pp. 8-23, 2011.

[62] F. K. Storm, W. H. Harrison, R. S. Elliott, and D. L. Morton, "Normal tissue and solid tumor effects of hyperthermia in animal models and clinical trials," Cancer Research, vol. 39, no. 6, Part 2, pp. 2245-2251, 1979. 
[63] X. Liang, H. Zhou, X. Liu et al., "Effect of local hyperthermia on lymphangiogenic factors VEGF-C and -D in a nude mouse xenograft model of tongue squamous cell carcinoma," Oral Oncology, vol. 46, no. 2, pp. 111-115, 2010.

[64] T. J. Vogl, P. Farshid, N. N. N. Naguib, and S. Zangos, "Thermal ablation therapies in patients with breast cancer liver metastases: a review," European Radiology, vol. 23, no. 3, pp. 797-804, 2013.

[65] C. S. Muenyi, A. R. Pinhas, T. W. Fan, G. N. Brock, C. W. Helm, and J. C. States, "Sodium arsenite \pm hyperthermia sensitizes p53-expressing human ovarian cancer cells to cisplatin by modulating platinum-DNA damage responses," Toxicological Sciences, vol. 127, no. 1, pp. 139-149, 2012.

[66] H. Ni, D. Zhang, N. Gu et al., "Preparation and characterization of $\mathrm{Mn}-\mathrm{Zn}$ ferrite magnetic nanoparticles for tumor hyperthermia," Journal of Chinese Electron Microscopy Society, vol. 25, no. 1, pp. 66-70, 2006.

[67] J. Liu, J. Zhang, L. Wang, Y. T. Li, and D. S. Zhang, "Biocompatibility study of $\mathrm{Mn}_{0.5} \mathrm{Zn}_{0.5} \mathrm{Fe}_{2} \mathrm{O}_{4}$ magnetic nanoparticles," Key Engineering Materials, vol. 483, pp. 552-558, 2011.

[68] C. Zhao, C. Yuan, and G. Wu, "p[5HRE]AFPp-p53/PEI-Fe $\mathrm{O}_{4}$ magnetic nanoparticle combined with magnetic fluid hyperthermia inhibit hepatoma cells," Chinese Journal of Cancer Biotherapy, vol. 23, no. 1, pp. 44-51, 2016.

[69] J. Xie, Y. Zhang, C. Yan et al., "High-performance PEGylated $\mathrm{Mn}-\mathrm{Zn}$ ferrite nanocrystals as a passive-targeted agent for magnetically induced cancer theranostics," Biomaterials, vol. 35, no. 33, pp. 9126-9136, 2014.

[70] O. K. Arriortua, E. Garaio, B. Herrero de la Parte et al., "Antitumor magnetic hyperthermia induced by RGD-functionalized $\mathrm{Fe}_{3} \mathrm{O}_{4}$ nanoparticles, in an experimental model of colorectal liver metastases," Beilstein Journal of Nanotechnology, vol. 7, pp. 1532-1542, 2016.

[71] M. Lin, J. Huang, X. Jiang et al., “A combination hepatomatargeted therapy based on nanotechnology: pHRE-Egr1HSV-TK/ ${ }^{131}$ I-antiAFPMcAb-GCV/MFH," Scientific Reports, vol. 6, no. 1, article 33524, 2016.

[72] Z. Y. Wang, J. Song, and D. S. Zhang, "Nanosized $\mathrm{As}_{2} \mathrm{O}_{3}$ / $\mathrm{Fe}_{2} \mathrm{O}_{3}$ complexes combined with magnetic fluid hyperthermia selectively target liver cancer cells," World Journal of Gastroenterology, vol. 15, no. 24, pp. 2995-3002, 2009. 


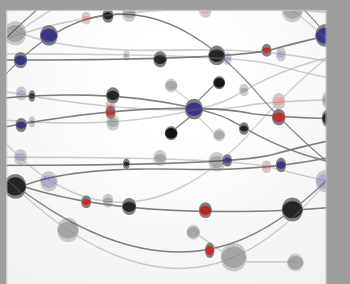

The Scientific World Journal
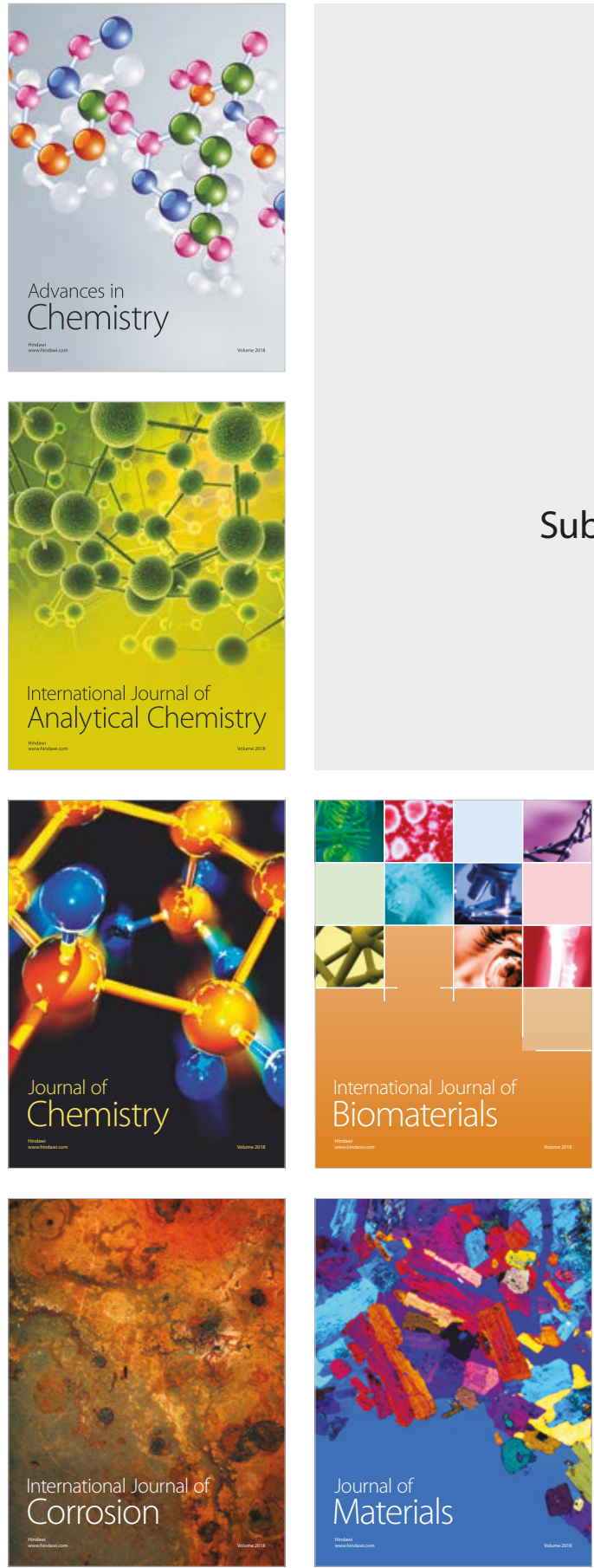

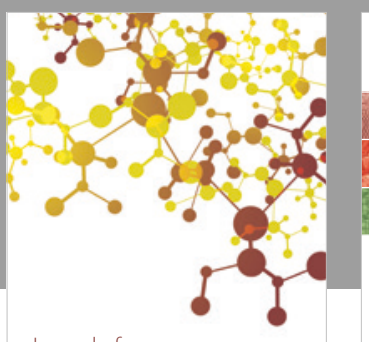

Journal of

Applied Chemistry
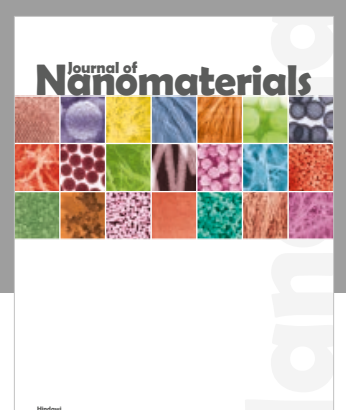

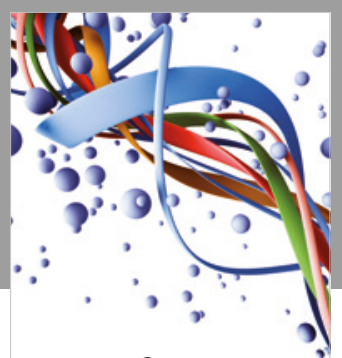

Scientifica

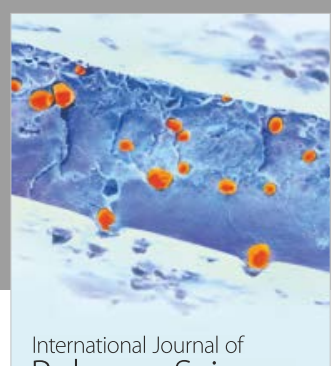

Polymer Science

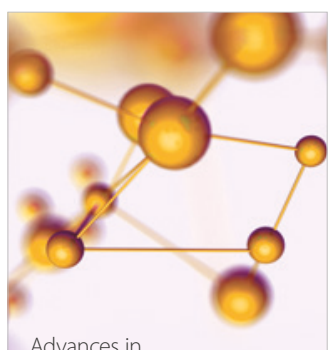

Physical Chemistry
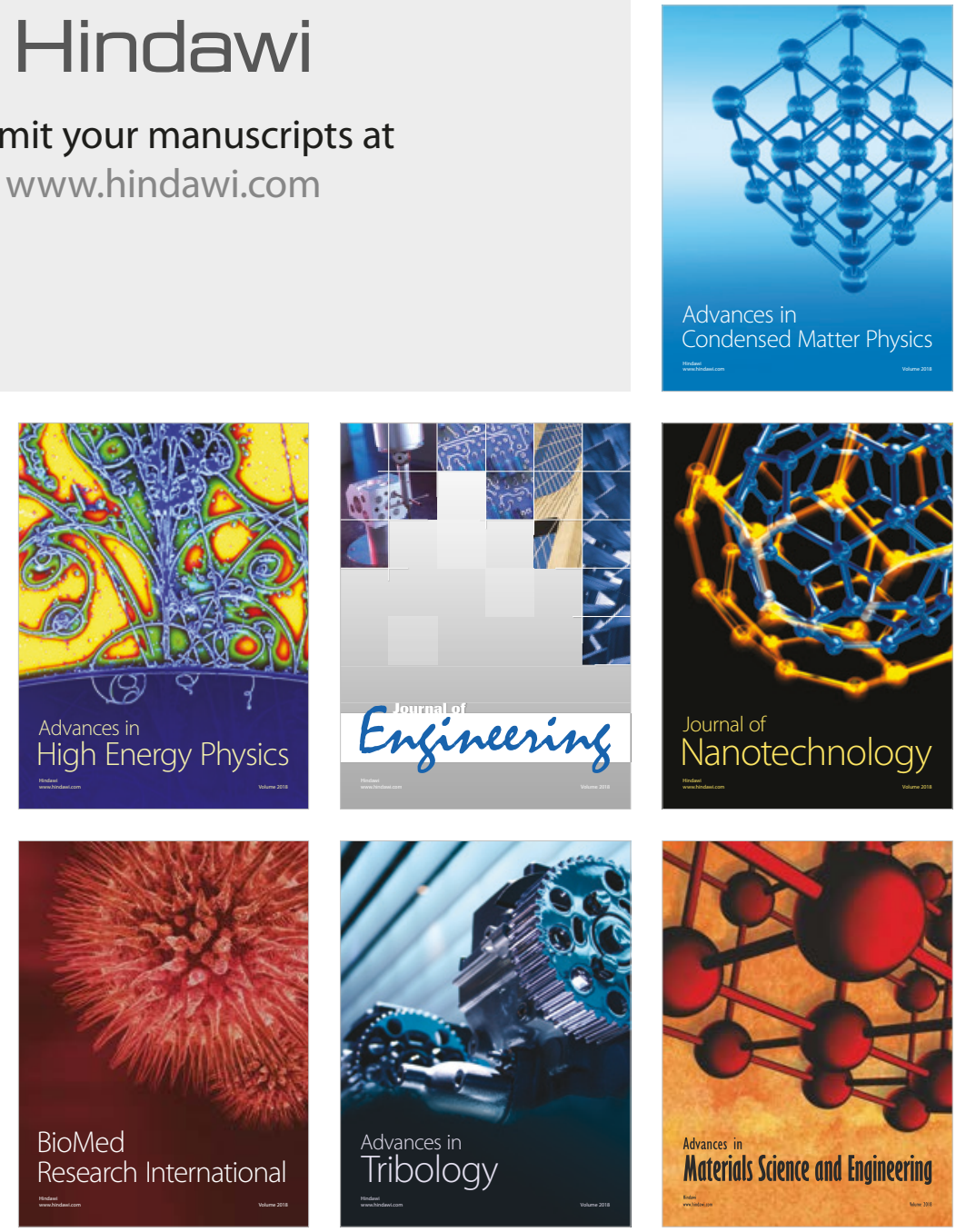